\title{
Diagnosis and treatment of severe asthma: a phenotype-based approach
}

\author{
Authors: Thomas L Jones, ${ }^{A^{*}}$ Daniel M Neville ${ }^{B^{*}}$ and Anoop J Chauhan ${ }^{C}$
}

\begin{abstract}
Severe asthma is a heterogeneous and often difficult to treat condition that results in a disproportionate cost to healthcare systems. Appropriate diagnosis and management of severe asthma is critical, as most asthma deaths have been retrospectively identified as having poorly recognised severe asthma. With multiple biologic agents becoming available, it is crucial to correctly phenotype patients in order to identify those that will respond to these high-cost treatments. We provide an overview of the assessment, phenotyping and management of severe asthma in primary and secondary care.
\end{abstract}

KEYWORDS: Assessment, asthma, phenotyping, treatment

\section{Introduction}

The label of severe asthma encompasses a heterogeneous group of patients who are managed on step $4-5$ of the British Thoracic Society (BTS) or Global Initiative for Asthma treatment ladder. ${ }^{1}$ Requiring high-dose inhaled steroid and multiple other controllers or oral corticosteroids to remain in control of their disease or uncontrolled despite this treatment, the burden of severe asthma to patients and to healthcare systems is high. Analysis from the BTS Difficult Asthma Registry in 2015 showed estimated annual treatment costs of $£ 2,912-£ 4,217$ per patient; this is driven by medication costs and, in those patients with frequent exacerbations, hospital admissions. ${ }^{2}$ In those with severe disease who are not receiving high-dose treatment, the cost can be even higher. In England and Wales alone, 1,302 people were recorded as dying of asthma in 2015, ${ }^{3}$ while the 2014 National Review of Asthma Deaths, commissioned by the Royal College of Physicians (RCP), identified deficiencies in care of two-thirds of the 195 cases of asthma mortality that they reviewed. ${ }^{4}$ This included underrecognition of disease severity by clinicians as well as patients and a lack of an asthma review in the preceding 12 months.

Authors: ${ }^{\text {A }}$ linical research fellow, Department of Respiratory Medicine, Portsmouth Hospitals NHS Trust, Portsmouth, UK; ${ }^{B}$ clinical research fellow, Department of Respiratory Medicine, Portsmouth Hospitals NHS Trust, Portsmouth, UK; C professor of respiratory medicine and consultant physician, Department of Respiratory Medicine, Portsmouth Hospitals NHS Trust, Portsmouth, UK; * joint first authors
With improved understanding of the heterogeneity of severe asthma and increasing treatment options available, those patients who are poorly responsive to treatment and who remain at high risk should be identified and be promptly referred to a specialist clinic. In this article, we aim to introduce a systematic approach and patient pathway for the assessment, phenotyping and management of severe asthma in non-specialist and specialist settings (Fig 1).

\section{Management by primary care / general physicians}

\section{Diagnosis}

The cardinal symptoms of asthma are dyspnoea, wheeze, cough and chest tightness. Symptoms are almost always variable and triggers, such as allergens or viral infections, should be identified and addressed where possible. Peak expiratory flow rate (PEFR) is a basic but valuable tool in the diagnosis and monitoring of asthma with demonstration of diurnal variable airflow obstruction suggestive of asthma and ongoing poor control.

Spirometry forms the mainstay of evaluation of airway disease, but good quality measurement is essential. Practitioners should be wary of results based on incomplete or non-reproducible manoeuvres as this can lead to over- or underdiagnosis. A lack of airway obstruction during symptoms should suggest alternative diagnoses although obstruction may not be present when well. Pre- and postbronchodilator spirometry allows assessment of reversibility - with an increase in forced expiratory volume in 1 second (FEV1) greater than $12 \%$ and $200 \mathrm{~mL}$ being suggestive of asthma, while greater than $400 \mathrm{~mL}$ practically confirms it. ${ }^{5} \mathrm{~A}$ lack of reversibility does not disprove asthma because chronic airway inflammation can cause structural remodelling and a reduction in bronchodilator response, or coexistent chronic obstructive pulmonary disease (COPD; known as Asthma-COPD overlap) can give a similar picture. Fractional exhaled nitric oxide (FeNO) is a measure of airway inflammation recommended by national guidelines, ${ }^{6}$ but is yet to be adopted for routine use in primary care. High levels of FeNO suggest ongoing 'type 2' airway inflammation, which supports the diagnosis of asthma and suggests responsiveness to corticosteroids. When detected in known asthmatics, it should provoke a discussion around a patient's compliance with treatment and/or prompt an increase in inhaled steroid therapy.

\section{Comorbidities and triggers}

Isolated severe asthma is rare; the majority of patients present with comorbidities and risk factors. Obesity, rhinosinusitis and 


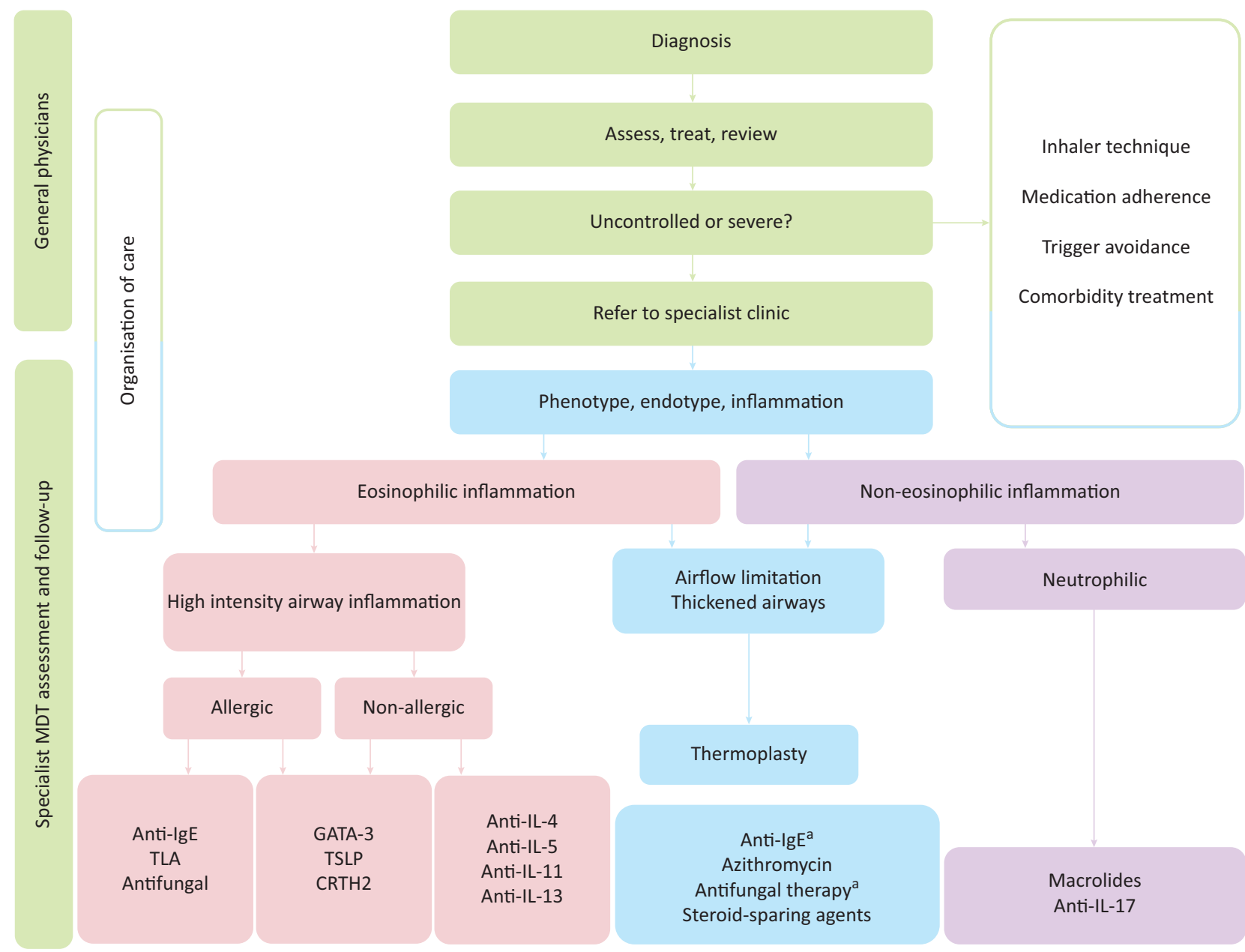

Fig 1. Management of severe asthma in primary and secondary care. ${ }^{a}$ for allergic disease with or without eosinophilia. CRTH2 = prostaglandin D2 receptor 2; $\mathrm{IgE}$ = immunoglobulin E; IL = interleukin; MDT = multidisciplinary team; MoAbs = monoclonal antibodies; TLA = temperature-controlled laminar airflow; TSLP = thymic stromal lymphopoetin

nasal polyposis, tobacco smoking, anxiety and depression, gastroesophageal reflux, breathing pattern disorders and osteopenia/osteoporosis are examples of comorbidities ${ }^{7}$ that result from the disease process or from treatment and add to the morbidity of asthma. Management of all of these factors will be familiar to the primary care practitioner and addressing each can make a large difference to a person's severe asthma or their perception of it.

The majority of patients with asthma will recognise triggers that worsen their symptoms; viral illnesses, temperature change or cold weather, allergen exposure and stress are common trigger factors. Patients will often not volunteer this information, so directed questioning is of use, particularly as patients will identify their triggers but never have considered avoiding them. Enquiring as to work-related symptoms is particularly important, as many patients are reticent to share difficulties with work for fear of endangering their occupation.

Management (inhaler technique, adherence and action plans)

With a multitude of inhaler devices available, it is vital that the correct inhaler type is prescribed for each patient; patient preference, manual dexterity and maximal inspiratory flow rate must be accounted for, particularly at the extremes of age and disease severity. Instruction on inhaler technique is often sadly lacking and most practitioners who treat asthma can relay anecdotes regarding the most inappropriate techniques that they have witnessed. Inhaler techniques are often counter-intuitive, particularly for patients on multiple inhaler device types: the slow steady inhalation of an metered-dose inhaler and the forced inhalation of a dry-powder inhaler can often be confused. Whether or not a patient's technique is correct, actual administration of the medication is another barrier; dispensing records may help identify patients who are less meticulous about their medication than they believe themselves to be and then development of an inhaler routine can frequently resolve symptoms. Progress in the production of 'smart inhalers', which record actuations and timings, may improve this as the bulk of non-adherence is likely unintentional and under-recognised by the patient.

Physiological measures often correlate poorly with patient symptoms in severe asthma. The use of patient reported outcome measures (PROMs) can help clinicians understand the burden of disease experienced by the patient and identify the significance of comorbidity when symptom burden outweighs 
airflow obstruction. Many PROMs are available, such as the Asthma Control Questionnaire, RCP's Three Questions and the Asthma Control Test. The evidence base for each PROM is variable $^{8}$ and their use should be limited to populations in which they have been validated; however, the use of PROMs is useful in the assessment and discussion of the multiple dimensions of a patient's disease.

Personalised asthma action plans have been shown to improve self-management of asthma ${ }^{9}$ and this is equally true in patients with severe disease. While PEFR-based management plans are appropriate in mild to moderate asthma, a drop in PEFR may be a late occurrence in severe asthma and increasing symptoms often precede PEFR changes by several days. Therefore, a symptom-based personalised asthma action plan may be advantageous in monitoring patients with severe asthma. If there is ongoing uncontrolled disease requiring frequent courses of oral corticosteroids or healthcare attendances, then timely assessment at a specialist clinic is warranted.

\section{Management in specialist clinic}

\section{Confirming the diagnosis}

On referral of a new patient to the severe asthma clinic, confirmation of the underlying diagnosis is usually the initial concern. Many patients with asthma have had the diagnosis made many years previously and the evidence on which it was made may or may not be strong. Further diagnostic tests available in specialist clinics include bronchodilator reversibility, challenge testing (mannitol, methacholine, histamine), exercise challenge and, to rule out alternative causes of breathlessness, cardiopulmonary exercise tests.

Management of comorbidity in the severe asthma population forms a large part of the workload of an asthma specialist in conjunction with a multidisciplinary team. Nasendoscopy, sinus computerised tomography, oesophageal manometry and impedance testing, dietary review, clinical psychology, specialist physiotherapy and speech and language therapy are all required and an essential part of the specialist asthma service. Failure to treat asthma-associated comorbidity will often preclude clinical improvement no matter how intensive the prescribed inhaled therapy is.

\section{Phenotype assessment}

As part of the investigative work-up, the specialist team will endeavour to phenotype every person's asthma. Severe asthma is now recognised as being a heterogeneous disease driven by multiple underlying pathobiological mechanisms ('endotypes'). The endotypes are not understood well enough for use in clinical medicine so phenotypes, collections of observable characteristics, are assessed instead. The use of phenotyping has been shown to improve management over symptom-based care alone. ${ }^{10}$ More recent opinion has suggested a move away from labelling of airways diseases and towards identification of phenotypic 'treatable traits' (such as airway eosinophilia and sinonasal disease) that improve symptoms when addressed. ${ }^{11}$

In order to assess the underlying pathobiology of a patient's asthma and target it appropriately, assessment of the airway inflammatory milieu is required. Although FeNO is known to correlate with airway eosinophilia, the association is imperfect; ${ }^{12}$ therefore, sputum induction is often performed to better understand the inflammatory process. This is achieved by nebulising saline of escalating tonicity. ${ }^{13}$ FEV1 monitoring is conducted throughout the procedure to ensure excess bronchoconstriction is avoided. The aim is to continue until expectoration of a sample of sputum; however, some patients never progress to this point. The sputum produced is then processed and the cellular content is counted - thresholds vary, but this gives a good impression of the overall level of eosinophilic and neutrophilic inflammation within the airways and can therefore guide appropriate treatment options.

\section{Eosinophilic asthma}

Eosinophilic asthma is driven primarily by type 2 cytokines. Previously known as Th2 when the predominant producer of these cytokines was thought to be type 2 T-helper cells, the discovery of type 2 innate lymphoid cells as a low prevalence but potent source of interleukin (IL)-4, 5, 13 and other type 2 cytokines led to a change in nomenclature.

Once confirmed, eosinophilic asthma may be considered allergic and non-allergic. Allergic disease fits the stereotype of asthma, often being early onset with an atopic background and high immunoglobulin $\mathrm{E}$ ( $\mathrm{IgE}$ ); aeroallergen exposure is considered one of the main drivers of inflammation in allergic asthma. ${ }^{14}$ Allergen avoidance advice is given as preventing exposure may reduce symptom burden; however, many aeroallergens - such as house dust mite or pollens - cannot easily be avoided. Omazliumab (Xolair) is a monoclonal antibody against IgE that acts to reduce the allergic response to allergens; ${ }^{15}$ it often has significant benefit in allergic asthma, but predicting who will or will not respond is not currently possible without a trial of treatment. ${ }^{16}$ Temperaturecontrolled laminar airflow (Airsonette, Airsonette AB, Sweden) is another upcoming treatment that is currently under study. It uses high efficiency particulate filtered air to reduce allergen exposure while the patient is in bed and would usually be exposed to high allergen loads. ${ }^{17}$

Non-allergic eosinophilic disease is often of later onset; IgE is frequently not elevated and nasal polyposis and salicylate sensitivity are more common. Multiple monoclonal antibodies are being produced to target 'type 2' high eosinophilic driven disease with mepolizumab being the first to be approved for use. Targeting IL-5 as the primary amplifying cytokine for eosinophils, mepolizumab (Nucala) provides another treatment option for nonallergic eosinophilic patients. ${ }^{18,19}$ Other upcoming treatments (and their cytokines) targeting eosinophilic disease include reslizumab (IL-5), ${ }^{20}$ benralizumab (IL-5 receptor) ${ }^{21}$ and dupilumab (IL-4/IL-13 receptor). ${ }^{22}$

\section{Non-eosinophilic asthma}

The non-eosinophilic (mainly neutrophilic) severe asthma phenotype is an increasingly recognised population that is poorly responsive to traditional escalating corticosteroid therapy. These patients have an apparent absence of type 2 driven inflammation and a neutrophilic predominance in their airways. ${ }^{23}$ Macrolide antibiotics have immunomodulatory and anti-inflammatory effects, and the use of 3-weekly azithromycin has been shown to reduce exacerbations and lower respiratory tract infections in noneosinophilic and eosinophilic asthmatics. ${ }^{24,25}$ Use of azithromycin is limited by the risk of hepatotoxicity and the requirement for regular blood monitoring; a recent large randomised controlled trial showed a lack of efficacy in acute exacerbations. ${ }^{26}$ IL-17 drives 
neutrophil recruitment and has been strongly correlated with neutrophilic severe asthma and steroid resistance. ${ }^{27,28}$ Despite having no effect on non-phenotyped severe asthma, brodalumab (IL-17 receptor monoclonal antibody) showed efficacy in a subgroup of patients with a high level of reversibility. ${ }^{29}$ Further development of therapies targeting non-eosinophilic asthma is ongoing and is much needed to increase treatment options for this subpopulation of people with severe asthma.

\section{Other treatments}

A recognised component of severe uncontrolled asthma is chronic inflammation that leads to increased airway smooth muscle mass with fixed airway thickening and airflow limitation. Bronchial thermoplasty is the delivery of controlled radiofrequency energy to the airway walls using a specially designed catheter. It has been shown to reduce airway smooth muscle mass, thereby decreasing the ability of the airways to constrict. ${ }^{30}$ Successive lobes are targeted in three separate treatment sessions, a minimum of 3 weeks apart; the right middle lobe is not treated and the upper lobes are treated in a single session. Bronchial thermoplasty has been shown to improve quality of life and reduce exacerbations in the year following treatment, with some evidence of sustained improved control up to 5 years. ${ }^{31}$

Immunosuppressive medications, such as ciclosporin and methotrexate, are in infrequent use, primarily as steroid sparing agents for patients requiring oral prednisolone at high doses. The evidence base for these immunosupressants is weak; ${ }^{32,33}$ on rare occasions they can have significant impact, but should be managed and monitored by a specialist multidisciplinary team.

A significant proportion of people with severe asthma are sensitised to fungi. Allergic bronchopulmonary aspergillosis is the best-known example of this, but more common is sensitisation without colonisation - known as severe asthma with fungal sensitisation. ${ }^{34}$ Identification of fungal sensitisation is important because patients can benefit from a trial of oral antifungal therapy. ${ }^{35}$

\section{Conclusions}

Severe asthma is a complex and heterogeneous disease that should be treated with a personalised approach. As mortality levels remain high, it is crucial that clinicians learn and respond to the deficiencies highlighted by the National Review of Asthma Deaths. ${ }^{4}$ Earlier recognition of severe asthma and a systematic approach to management increases the opportunity for targeted treatment, preventing disease progression and leading to improved outcomes.

\section{Conflicts of interest}

The authors have no conflicts of interest to declare.

\section{References}

1 Chung KF, Wenzel SE, Brozek JL et al. International ERS/ATS guidelines on definition, evaluation and treatment of severe asthma. Eur Respir ] 2014:43:343-73.

2 O'Neill S, Sweeney J, Patterson CC et al. The cost of treating severe refractory asthma in the UK: an economic analysis from the British Thoracic Society Difficult Asthma Registry. Thorax 2015;70:376-8.
3 Office of National Statistics. Asthma deaths in England and Wales, 2001 to 2015 occurrences. Newport: ONS, 2016. www.ons.gov. uk/peoplepopulationandcommunity/birthsdeathsandmarriages/ deaths/adhocs/005955asthmadeathsinenglandandwales2001 to2 015occurrences [Accessed 4 September 2017].

4 Levy ML, Andrews R, Buckingham R et al. Why asthma still kills: the National Review of Asthma Deaths (NRAD). London: Royal College of Physicians, 2014.

5 British Thoracic Society, Scottish Intercollegiate Guidelines Network. British guideline on the management of asthma. SIGN guideline No 153. Edinburgh: SIGN, 2016.

6 National Institute for Health and Care Excellence. Measuring fractional exhaled nitric oxide concentration in asthma: NIOX MINO, NIOX VERO and NObreath. NICE diagnostics guideline No 12. London: NICE, 2014.

7 Sweeney J, Patterson C, Menzies-Gow A et al. Comorbidity in severe asthma requiring systemic corticosteroid therapy: cross-sectional data from the Optimum Patient Care Research Database and the British Thoracic Difficult Asthma Registry. Thorax 2016;71:339-46.

8 Worth A, Hammersley V, Knibb R et al. Patient-reported outcome measures for asthma: a systematic review. NPJ Prim Care Respir Med 2014:24:14020.

9 Gibson PG, Powell H. Written action plans for asthma: an evidencebased review of the key components. Thorax 2004:59:94-9.

10 Green RH, Brightling CE, McKenna S et al. Asthma exacerbations and sputum eosinophil counts: a randomised controlled trial. Lancet 2002;360:1715-21.

11 Agusti A, Bel E, Thomas M et al. Treatable traits: toward precision medicine of chronic airway diseases. Eur Respir ] 2016;47:410-9.

12 Westerhof GA, Korevaar DA, Amelink M et al. Biomarkers to identify sputum eosinophilia in different adult asthma phenotypes. Eur Respir ] 2015;46:688-96.

13 Djukanović R, Sterk PJ, Fahy JV, Hargreave FE. Standardised methodology of sputum induction and processing. Eur Respir J Suppl 2002;37:1s-2s.

14 Baxi SN, Phipatanakul W. The role of allergen exposure and avoidance in asthma. Adolesc Med State Art Rev 2010;21:57-71.

15 Hanania NA, Alpan O, Hamilos DL et al. Omalizumab in severe allergic asthma inadequately controlled with standard therapy: a randomized trial. Ann Intern Med 2011;154:573-82.

16 National Institute for Health and Care Excellence. Omalizumab for treating severe persistent allergic asthma. NICE technology appraisal guideline No 278. London: NICE, 2013.

17 Boyle RJ, Pedroletti C, Wickman M et al. Nocturnal temperature controlled laminar airflow for treating atopic asthma: a randomised controlled trial. Thorax 2012;67:215-21.

18 Pavord ID, Korn S, Howarth P et al. Mepolizumab for severe eosinophilic asthma (DREAM): a multicentre, double-blind, placebocontrolled trial. Lancet 2012;380:651-9.

19 National Institute for Health and Care Excellence. Mepolizumab for treating severe refractory eosinophilic asthma. NICE technology appraisal guideline No 431. London: NICE, 2017.

20 Castro M, Mathur S, Hargreave F et al. Reslizumab for poorly controlled, eosinophilic asthma: A randomized, placebo-controlled study. Am J Respir Crit Care Med 2011;184:1125-32.

21 Laviolette M, Gossage DL, Gauvreau G et al. Effects of benralizumab on airway eosinophils in asthmatic patients with sputum eosinophilia. J Allergy Clin Immunol 2013;1321086-96.e5.

22 Wenzel S, Ford L, Pearlman D et al. Dupilumab in persistent asthma with elevated eosinophil levels. N Engl J Med 2013;368:2455-66.

23 Moore WC, Bleecker ER, Curran-Everett D et al. Characterization of the severe asthma phenotype by the National Heart, Lung, and Blood Institute's Severe Asthma Research Program. J Allergy Clin Immunol 2007;119:405-13.

24 Brusselle GG, VanderStichele C, Jordens P, Deman R, Slabbynck H. Azithromycin for prevention of exacerbations in severe asthma 
(AZISAST): a multicentre randomised double-blind placebo-controlled trial. Thorax 2013;68:322-9.

25 Gibson PG, Yang IA, Upham JW et al. Effect of azithromycin on asthma exacerbations and quality of life in adults with persistent uncontrolled asthma (AMAZES): a randomised, double-blind, placebo-controlled trial. Lancet 2017;390:659-68.

26 Johnston SL, Szigeti M, Cross M et al. Azithromycin for acute exacerbations of asthma: the AZALEA randomized clinical trial. JAMA Intern Med 2016;176:1630-7.

27 Chesne J, Braza F, Mahay G et al. IL-17 in severe asthma: where do we stand? Am J Respir Crit Care Med 2014;190:1094-101.

28 Agache I, Ciobanu C, Agache C, Anghel M. Increased serum IL-17 is an independent risk factor for severe asthma. Respir Med 2010;104:1131-7.

29 Busse WW, Holgate S, Kerwin E et al. Randomized, double-blind, placebo-controlled study of brodalumab, a human anti-IL-17 receptor monoclonal antibody, in moderate to severe asthma. Am J Respir Crit Care Med 2013;188:1294-302.

30 National Institute for Health and Care Excellence. Bronchial thermoplasty for severe asthma. NICE interventional procedures guideline No 419. Manchester: NICE, 2012.
31 Torrego A, Solà I, Munoz A et al. Bronchial thermoplasty for moderate or severe persistent asthma in adults. Cochrane Database Syst Rev 2014;(3):CD009910.

32 Davies HRHR, Olson LLG, Gibson PG. Methotrexate as a steroid sparing agent for asthma in adults. Cochrane Database Syst Rev 1998;(3):CD000391.

33 Evans D], Cullinan P, Geddes DM et al. Cyclosporin as an oral corticosteroid sparing agent in stable asthma. Cochrane Database Syst Rev 2000;(4):CD002993.

34 Denning DW, O'Driscoll BR, Hogaboam CM, Bowyer P, Niven RM. The link between fungi and severe asthma: a summary of the evidence. Eur Resp J 2006;27:615-26.

35 Parulekar AD, Diamant Z, Hanania NA. Antifungals in severe asthma. Curr Opin Pulm Med 2015;21:48-54.

Address for correspondence: Dr Thomas Jones, Department of Respiratory Medicine, Portsmouth Hospitals NHS Trust, Portsmouth, Hampshire PO6 3LY, UK.

Email: Tom.jones@doctors.org.uk 